\section{Survival, Reasons for Failure and Clinical Characteristics of Anterior/ Posterior Composites: 8-Year Findings}

Anelise Fernandes Montagner ${ }^{1}$, Francoise Helene van de Sande ${ }^{2}$, Clecila Müller ${ }^{1}$, Maximiliano Sérgio Cenci $^{2}$, Alexandre Henrique Susin ${ }^{1}$
'Graduate Program in Dentistry, UFSM - Universidade Federal de Santa Maria, Santa Maria, RS, Brazil ${ }^{2}$ Graduate Program in Dentistry, UFPel - Universidade Federal de Pelotas, Pelotas, RS, Brazil

Correspondence: Anelise Fernandes Montagner, Rua Floriano Peixoto, 1184, 97015-372 Santa Maria, RS, Brazil. Tel.: +55-55-99124-7829. e-mail: animontag@gmail.com

\begin{abstract}
This retrospective study aimed to compare the annual failure rate (AFR), reasons for failure and factors influencing survival of posterior and anterior composite restorations placed by undergraduate students. Composite restorations placed by undergraduate students were evaluated. The restorations should be in occlusion, with at least one adjacent tooth; and patients should have been present for check-up, with at least 1 annual recall. The investigation was performed in two separate analysis: 1) dental electronic records of 100 patients ( $n=333$ restorations) were selected to assess factors influencing survival; 2 ) clinical evaluation of 30 patients by examining restorations $(n=123)$ to assess clinical characteristics and failure type distribution. Data were subjected to Kaplan-Meier method, Log-rank test, Cox regression and Fisher's Exact test $(p<0.05)$. After 8 -year period, the AFR was $2.62 \%$. There was difference between anterior and posterior restorations $(p=0.005)$, with anterior showing a higher AFR. The patient caries-risk $(p<0.001)$ and the number of surfaces restored $(\mathrm{p}=0.010)$ affected restoration survival. The superficial brightness $(p=0.029)$, fracture $(p=0.025)$ and retention $(p=0.011)$ were clinical criteria with differences between anterior and posterior restorations. Income $(p<0.001)$, caries activity $(p<0.001)$, caries risk $(p<0.001)$ and oclusal risk $(p<0.001)$ also influenced on the restorations survival. After 8-year, the AFR of restorations placed by undergraduate students was acceptable and affected by patient risk factors and the position of the tooth in the arch: anterior restorations failed more than posterior.
\end{abstract}

Key Words: clinical study, dental restoration failure, longevity, survival analysis, risk factors.

\section{Introduction}

Composite resin is regarded as a suitable restorative material for direct restorations in anterior and posterior teeth, both in permanent and deciduous dentitions, presenting high survival rates and acceptable clinical performance in studies with long-term follow-ups (1-3). Even with this successful survival rates, a considerable proportion of time of dentists in dental practice and of students in dental schools is devoted to replacing restorations. The incentive to replace or to repair those restorations is frequently based on preventing or mitigating secondary caries lesions, both in an attempt to identify the caries lesion itself and to restore marginal defects that may favour lesion development (4).

Data on the longevity of composite restorations in posterior teeth have been widely explored. The most common reasons for restoration failure are caries development and fracture in posterior teeth, with secondary caries as the main cause of composite restoration failures in high caries-risk patients $(5,6)$. This has wide-reaching implications for the longevity of affected teeth and health expenditure. The use of composite resin is widespread also in anterior restorations; however, data on the longevity of composite restorations in anterior teeth is still limited in literature. In anterior teeth, the survival rates has been reported even higher than in posterior teeth, but the main cause of composite restoration failures is concerning to aesthetic reasons (7).

It was shown that the longevity of restorations increased for those placed by more experienced clinicians (8). Besides all available evidence on survival of composite restorations, most studies have been performed in a specific controlled scenario. Most data on longevity of composite restorations available in the literature are from studies in which experienced and trained dentists placed the restorations in low-risk patients with a high social-economic level (1). Thus, the considered annual failure rate could not be extrapolated as a true reality. In most retrospective studies, the scenario is made in dental practices with experienced dentists as operators, and in low-risk patients $(1,7)$. Moreover, in controlled clinical trials, restorations are often placed by experienced and extensively trained operators, following strict inclusion criteria, according to rigorous and calibrated conditions (9). In controlled clinical trials, annual failure rates of posterior composite resin restorations are estimated and ranged between 0 and $9 \%$ after 5 years (10). Further, considering the restrictions to conduct randomized controlled trials, data on restoration survival obtained from 
retrospective clinical studies working with larger group of patients are being successful performed since they require lower cost and are more suited for studying the survival of a significant amount of restoration for a long period of time $(1,7)$.

Data on the survival rate of resin composite restorations placed by undergraduate students in dental schools are still rare. As students are note experienced, the longevity of composite resin restorations may be disadvantaged in dental schools since students must to develop their skills during the course (6). It was reported that dental students are able to place resin composite restorations in posterior teeth with an acceptable mean annual failure rate, however the scarce studies with inexperienced operators showed higher annual failure rates $(1.7 \%$ to 2.8$)(8,11)$ compared to experienced dentists (1.0\% to $1.5 \%)(1)$.

At dental schools worldwide, the teaching of posterior and anterior composites restorations is a well-established element of undergraduate dental students and therapy training $(11,12)$. Thus, considering the trend towards the use of direct composite resin for restoration and the lack of evidence on the survival rate of resin composite restorations placed by students, the aim of this retrospective study was to compare the annual failure rate (AFR), reasons for failure and factors influencing survival of posterior and anterior composite resin restorations placed by undergraduate students over an eight-year follow-up period.

\section{Material and Methods}

Study Design

This was a double-blind (examiners and statistician) study based on a retrospective longitudinal evaluation of posterior and anterior composite restorations placed by dental students (2nd up to 5th year) in a Public School of Dentistry (Federal University of Santa Maria - UFSM, Santa Maria, RS, Brazil). The survival of restorations up to 8 years follow-up was determined, and factors potentially associated with failure were investigated. Data were collected in two ways: 1) the history of each restoration was extracted from the dental electronic records and 2) from clinical examinations. For the clinical evaluation, the patients were invited to participate in the study by telephone contact by a researcher involved in the project (AFM). Direct composite resin restorations, with at least 4 years, in anterior and posterior teeth performed by dental students were clinically evaluated by two calibrated examiners based on the criteria proposed by FDI $(13,14)$.

\section{Ethical Aspects}

This study was approved by the local Ethics Committee (Nr. 40246314.3.0000.5346) and followed STROBE guidelines (15). The patients enrolled in the selected records were contacted by telephone and invited to participate as volunteer and come to School of Dentistry (UFSM) for clinical evaluation of restorations. For clinical examinations, prior to the participation in the study, all participants signed a written informed consent after being informed about the purposes of the study. The dental needs of the subjects enrolled in this study were provided by the undergraduate dental students, under supervision, during the whole study period, except for prosthetic rehabilitation and orthodontic treatment.

\section{Dental Records Assessment}

Patient records from the public Scholl of Dentistry (Federal University of Santa Maria, RS, Brasil) were used for data collection and patient's selection. One hundred (100) electronic dental records were randomly selected in the Dental Record Manager (an existing electronic database with patient files) of the Dental School. The randomization was carried out in a computer program (Microsoft Excel-2010) and a randomization table was used to search for 100 dental records that would full-fill the eligibility criteria by random numbers. An excel table with random numbers from 1 up to 8,000 was generated. If the patient does not fit the inclusion criteria, the next number was considered, and so on. One person not directly involved with the study prepared this random table in advance.

Dental electronic records of patients who attended the dental practice from January 2007 to December 2012 and received at least one composite resin restoration were searched for eligibility according to the following inclusion criteria: (a) the restorations should be in occlusion, with at least one adjacent tooth; and (b) patients should have been present for check-up or follow-up treatment, with at least 1 annual recall.

An experienced researcher collected all the records data. The history of each restoration was extracted from the dental electronic records. All the dental procedures performed at the Dental School (Federal University of Santa Maria, RS, Brasil) are described in details at the electronic record. This Dental Manager that store the dental records was developed in 2007 and currently contains all data relating to patients, students and the procedures performed in the Disciplines of Restorative Dentistry II ( $2^{\text {nd }}$ year), Restorative Dentistry III ( $3^{\text {rd }}$ year), and the Clinical Integrated Units I up to IV ( $3^{\text {rd }}$ up to $5^{\text {th }}$ year). All procedures performed by undergraduate students in the clinics at Dental School are under the supervision of a tutor (professor). The restorative procedures were performed under rubber dam isolation.

The data collected were: the operator experience (year that the student was in the course), the caries risk (considering the past dental history), the gender, date of 
the restorative procedure and details (brand of composite resin, number of faces involved in the restoration, shade of restoration, adhesive system used, presence of endodontic treatment and presence of liner), and data of the recall visits.

\section{Clinical Assessment}

At the dental records there was no information referring to the clinical characteristics and types of failures, therefore, the clinical evaluation was performed. After the dental records search, the recruitment of patients for clinical assessment was performed by phone calls, from March to August 2015, when the patients visited the Dental School for a check-up, were invited to visit the dental Scholl for evaluation. Thus, all patients included in the dental records assessment were invited for the clinical examination. The inclusion criteria were: (a) patients aged 18 or older, (b) patients enrolled in the Dental Manager System with address in Santa Maria, (c) patients who received direct resin composite restorations in anterior and / or posterior teeth performed by students from 2007 to 2012 UFSM, (d) patients able to understand and sign the free and informed consent, (e) and patients willing to return to revaluation queries. The exclusion criteria were: (a) tooth restored without presence of antagonist tooth, (b) patients in orthodontic treatment, (c) patients that did not attend for check-up or follow-up treatment, with at least 1 annual recall, (d) patients that reported dental interventions in others dental practice, (e) and patients with impaired general health condition.

Criteria approved by the FDI World Dental Federation were used for clinical assessment of restorations $(13,14)$. The primary clinical outcome was restoration retention, considering as failure the loss of restoration. Secondary endpoints included the criteria as follows: 1) marginal adaptation, 2) marginal staining, 3) surface staining, 4) postoperative sensitivity, 5) superficial brightness, 6) translucency and color, 7) fracture, 8) anatomic form and 9) preservation of tooth vitality and integrity. Each criteria was expressed in five scores, three for acceptable and two for non-acceptable (one for reparable and one for replacement). Restorations that needed replacements were considered clinical failures (score 5).

Two blinded and calibrated examiners (AFM and AHS) carried out the evaluations independently; surfaces were air-dried and examined by means of an explorer and dental mirror. In cases of disagreement, a third evaluation was undertaken with both examiners until they reached consensus. The examiners were blinded to the interventions. A web-based training and calibration tool (www.ecalib. info) and clinical setting evaluation were used for training and calibration of the examiners. The clinical intra- interexaminers calibration was carried out with 40 composite restorations (20 anterior and 20 posterior restorations).
A pre-evaluation intra- inter-agreement of at least 90\% was obtained. The clinical evaluation was performed March up to August 2015, according to FDI criteria (14), evaluating esthetic, functional, and biological properties of the restorations in situ.

During the clinical evaluation of the restorations, patients were asked to answer questions related to socioeconomic factors, income, gender, and age. They were assessed for caries activity (based on the presence of active caries lesions) and caries risk. A classification of caries risk status was based on past dental history and was estimated based on previous reports $(5,16,17)$ in order to qualitatively assess the risk according to simplified criteria: after 3 years of restoration placement, the patient was considered as "high-caries-risk" if more than one new caries lesion was detected; and the patient was considered as "low-cariesrisk" in other cases. They were also assessed for occlusal stress factors based on a previous report (5). Patient risk estimation concerning bruxism/parafunctional habits was determined by self-report $(5,18)$ of five specific questions and clinical examination for specific clinical parameters (presence of: a. facets parallel to the normal planes of contour; b. noticeable flattening of cusps or incisal edges; and c. total loss of contour and dentinal exposure when identifiable). If the patient answered positively on 2 or more of the 6 questions and presented one of the clinical parameters, they were classified as "high-occlusal-stressrisk". In other cases, they were classified as "low-occlusalstress-risk".

\section{Outcomes}

The primary endpoint considered was the failure of the restoration, ie, the need for repair or replacement of restoration. The cause of the failure of the restoration was recorded. Secondary outcomes for clinical assessment were: 1) patient's caries risk, 2) patient's occlusal risk, 3) caries activity, 4) income, 5) age, 6) gender, 7) FDI criteria: marginal adaptation, marginal staining, surface staining, postoperative sensitivity, superficial brightness, translucency and color, fracture, anatomic form and preservation of tooth vitality and integrity.

\section{Statistical Analysis}

The data were submitted to statistical analysis, considering $80 \%$ statistic power and 5\% significance level. Statistical analysis was carried out using the SPSS 20.0 statistical package (SPSS Inc., Chicago, IL, USA). The analysis was performed in two separate parts: 1) Dental Records Analysis: data were subjected to survival analysis using the Kaplan-Meier method. The log-rank test was used to evaluate the existence of differences between the survival curves (anterior $\mathrm{x}$ posterior restorations). 
Moreover, a Cox regression was applied on the data to evaluate the influence of the variables on the restorations survival over time $(p<0.05)$, estimating the Hazard Ratios (HR) and 95\% confidence intervals. 2) Clinical Assessment Analysis: qualitative analysis based on the FDI criteria was analyzed independently for each of the evaluated clinical characteristics. Differences in these qualitative criteria between anterior and posterior restorations were analyzed using Fisher's Exact test $(p<0.05)$. A Cox regression was applied on the data to evaluate the influence of the variables on the restorations survival over time $(p<0.05)$, estimating the Hazard Ratios (HR) and 95\% confidence intervals.

\section{Results}

\section{Dental Records Assessment}

A total of 333 of composite restorations (143 anterior e 190 posterior) were evaluated in dental records of 100 patients. The Kaplan Mayer test showed that after 8-year period, a total of $21 \%$ of the restorations failed (the anterior restorations failed more than the posterior) showing an annual failure rate of 2.6. The failures after 8-year occurred in 40 anterior and 30 posterior restorations (Table 1). The annual failure rate for anterior restorations was 3.49\% and for posterior was 1.95\%. The majority of failed events occurred after 3 years and before 6 years (Fig. 1).

The Log-Rank test showed a difference in the comparisons of annual survival rates between anterior and posterior restorations groups ( $p=0.005)$.

Cox regression showed that the different variables influence on the restorations survival, as the position of the tooth in anterior or posterior segment $(p=0.005)$, the patient caries risk $(p<0.001)$, and when the restorations have 3 or more surfaces restored ( $p=0.01)$. The results for the Cox regression test after 8-year period of dental records are present in Table 2.

\section{Clinical Assessment}

Clinical evaluations were performed in 30\% of the selected electronic records sample. A total of 123 restorations (78 anterior and 45 posterior) placed by undergraduate students in 30 patients ( 14 women and 16 man) were clinically evaluated. The patients were adults with a mean age of 55 years old (minimum 21 and maximum 76 years). Regarding the socioeconomic status, most participants came from low-income families $( \pm \cup \$$ 500.00, two minimum monthly Brazilian wage).

The Fisher's Exact Test showed that there was statistically significance on the scores for anterior and posterior restorations $(p=0.02)$. The comparisons between the anterior and posterior restorations, according each FDI criteria are present in Table 3. After 8-year of follow up most restorations presented a FDI score 1 or 2 (clinically acceptable). The superficial brightness $(p=0.029)$, fracture $(p=0.025)$ and retention $(p=0.011)$ were clinical criteria with differences between anterior and posterior restorations, with anterior restoration with higher scores than posterior. After 8-year of follow-up, 33 restorations failed. The mains causes for failures were secondary caries and fracture for posterior restorations and fracture and aesthetic reasons for anterior restorations.

The Cox Regression analysis showed that income $(p<0.001, H R 0.26, C l 0.16-0.42)$, caries activity $(p<0.001$, $\mathrm{HR} 3.52, \mathrm{Cl} 2.10-5.87$ ), caries risk ( $<<0.001, \mathrm{HR} 2.67, \mathrm{Cl}$ $1.83-3.54)$, oclusal risk ( $<<0.001, \mathrm{HR} 1.94, \mathrm{Cl} 1.10-2.98)$ were factors that statistically influenced on the restorations survival. The other factors, as age and gender (all $p>0.05$ ),

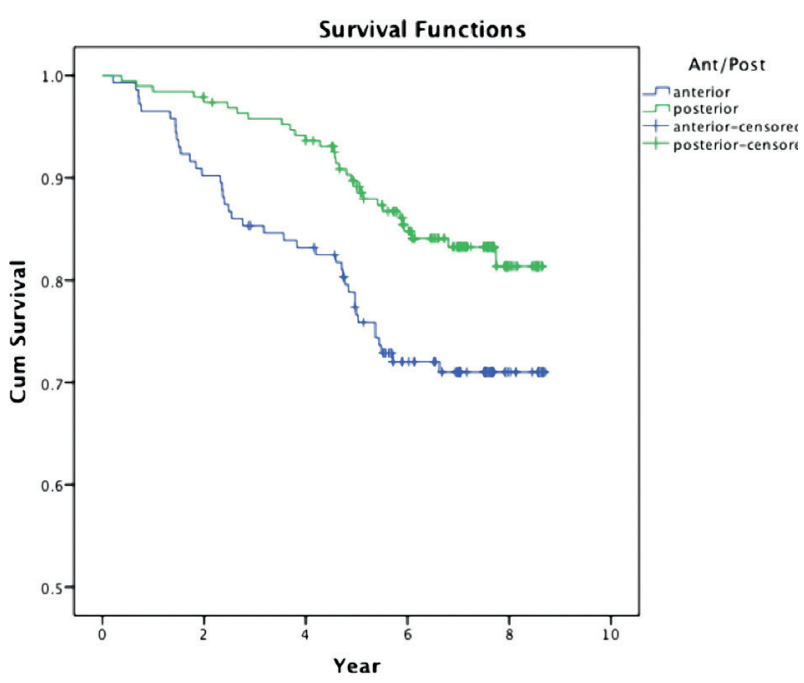

Figure 1. Survival curves (Kaplan-Meier) for anterior and posterior restorations over the 8-year observation period for the dental records.

Table 1. Survival characteristics of dental records

\begin{tabular}{lccccc}
\hline Group & Number of restorations & Number of failures & Number of censored & $\begin{array}{c}\text { Mean survival time } \\
\text { in years (95\% CI) }\end{array}$ & $\begin{array}{c}\text { Annual failure } \\
\text { rate } \% \text { at 8-year }\end{array}$ \\
\hline Anterior & 143 & 40 & $103(72.0 \%)$ & $7.12(6.68-7.56)$ & $3.49 \%$ \\
Posterior & 190 & 30 & $160(84.2 \%)$ & $7.90(7.62-8.15)$ & $1.95 \%$ \\
Overall & 333 & 70 & $263(78.9 \%)$ & $7.58(7.34-7.83)$ & $2.62 \%$ \\
\hline
\end{tabular}


did not significantly affect on the restorations survival after 8-year period.

\section{Discussion}

The present study showed that there was a difference in

Table 2. Results for the Cox regression for the 8-year period of dental records data

\begin{tabular}{|c|c|c|c|c|}
\hline \multirow{2}{*}{ Variable } & \multirow{2}{*}{ p-value } & \multirow{2}{*}{$\begin{array}{l}\text { Hazard } \\
\text { Ratio } \\
\text { Exp (b) }\end{array}$} & \multicolumn{2}{|c|}{$\begin{array}{c}\text { Confidence } \\
\text { interval }\end{array}$} \\
\hline & & & Lower & Upper \\
\hline \multicolumn{5}{|l|}{ Tooth Type } \\
\hline Anterior / Posterior & $p=0.005$ & 0.42 & 0.23 & 0.76 \\
\hline Superior / Inferior & $p=0.76$ & 1.09 & 0.61 & 1.95 \\
\hline Presence of Liner & $\mathrm{p}=0.18$ & 0.57 & 0.25 & 1.29 \\
\hline Endodontic treatment & $p=0.60$ & 1.26 & 0.52 & 3.02 \\
\hline Type of resin & $p=0.46$ & 0.46 & 0.06 & 3.61 \\
\hline Caries Risk & $\mathrm{p}<0.001$ & 13.96 & 4.28 & 45.55 \\
\hline \multicolumn{5}{|l|}{ Number of surfaces } \\
\hline$\geq 3$ surfaces & $p=0.01$ & 1 & 1 & 1 \\
\hline$=2$ surfaces & $p=0.12$ & 1.75 & 0.86 & 3.56 \\
\hline$=1$ surface & $p=0.29$ & 0.68 & 0.34 & 1.38 \\
\hline \multicolumn{5}{|l|}{ Student Experience } \\
\hline 2nd year & $p=0.97$ & 1 & 1 & 1 \\
\hline 3rd year & $p=0.81$ & 1.12 & 0.30 & 4.72 \\
\hline 4 th year & $\mathrm{p}=0.42$ & 0.58 & 0.16 & 2.19 \\
\hline 5th year & $p=0.46$ & 1.59 & 0.43 & 5.48 \\
\hline
\end{tabular}

survival rate of anterior and posterior restorations placed by students of a public university in the South of Brasil (UFSM), however the experience of the students did not influence on the restoration survival. The annual failure rate in this study was $2.6 \%$, a higher annual failure rate than that showed in previous prospective studies performed by experienced operators in dental practices (1 up to $2 \%)(1,3,9)$. Besides to consider the different operator's experience (undergraduate students vs. experience dentist), it should be considered also the profile of the patients. At the Dental School of UFSM, most of the patients are high-risk patients considering the caries experience, the educational level and the low socioeconomic level. The survival of restorations has shown to be affected by patient risk factors, mainly the caries and oclusal risk factors (5), thus focusing on the influence of those potential factors has been highlighted. In the present study, the caries risk was a factor that considerably increased the chance of restoration failure. The present findings may reflect the clinical reality of patients with low income that have no access to private dental clinics, and it can be inferred that these patients undergone less dental visits than the patients that have higher income.

The difference in longevity between dental faculty and general practitioners has been reported (19). An explanation for this findings is that the operator experience is another factor that has shown to play a role in the restoration survival (8). However, in the present study the undergraduate student's experience (based on the years of studying/practicing) did not influence the restoration survival, which suggests that maybe the operator's ability could be more important than the operator's experience. Conversely, the operator's ability to make restorations could

Table 3. Comparison between the anterior $(n=78)$ and posterior $(n=45)$ restorations, according to the FDI criteria compared by Fisher's Exact Test at $\mathrm{p}<0.05$. Numbers separated by slash represent the number of evaluated restorations for each score, according to the FDI criteria: 1 . Clinically excellent; 2. Clinically good; 3. Clinically sufficient / satisfactory; 4. Clinically unsatisfactory; 5 . Clinically poor

\begin{tabular}{|c|c|c|c|c|}
\hline \multirow{2}{*}{$\begin{array}{l}\text { General } \\
\text { evaluated criteria }\end{array}$} & \multirow[b]{2}{*}{ Specific evaluated criteria } & Anterior Restorations & Posterior Restorations & \multirow[b]{2}{*}{$\mathrm{p}$ value } \\
\hline & & $\begin{array}{l}\text { Restoration within each } \\
\text { score }(1 / 2 / 3 / 4 / 5)\end{array}$ & $\begin{array}{l}\text { Restoration within each } \\
\text { score }(1 / 2 / 3 / 4 / 5)\end{array}$ & \\
\hline \multirow{5}{*}{ Esthetics properties } & Superficial brightness & $18 / 45 / 13 / 2 / 0$ & $8 / 18 / 19 / 0 / 0$ & $\mathrm{p}=0.029$ \\
\hline & Surface staining & $18 / 47 / 11 / 2 / 0$ & $7 / 27 / 9 / 1 / 1$ & $p=0.589$ \\
\hline & Marginal staining & $18 / 32 / 21 / 4 / 3$ & $10 / 11 / 21 / 3 / 0$ & $\mathrm{p}=0.104$ \\
\hline & Translucency and color Stability & $19 / 43 / 14 / 2 / 0$ & $8 / 29 / 7 / 1 / 0$ & $\mathrm{p}=0.873$ \\
\hline & Anatomic form & $17 / 53 / 5 / 1 / 2$ & $9 / 27 / 5 / 4 / 0$ & $\mathrm{p}=0.619$ \\
\hline \multirow{4}{*}{ Functional properties } & Fracture & $54 / 3 / 2 / 0 / 19$ & $27 / 8 / 2 / 3 / 5$ & $p=0.025$ \\
\hline & Retention & $18 / 56 / 1 / 0 / 3$ & $8 / 30 / 7 / 0 / 0$ & $\mathrm{p}=0.011$ \\
\hline & Marginal adaptation & $18 / 45 / 10 / 3 / 2$ & $7 / 27 / 8 / 2 / 1$ & $p=0.795$ \\
\hline & Patient perception & $59 / 18 / 1 / 0 / 0$ & $37 / 7 / 1 / 0 / 0$ & $\mathrm{p}=0.556$ \\
\hline \multirow{3}{*}{ Biological properties } & Postoperative sensitivity & $55 / 19 / 2 / 1 / 1$ & $32 / 8 / 2 / 1 / 2$ & $\mathrm{p}=0.216$ \\
\hline & Teeth integrity & $60 / 18 / 0 / 0 / 0$ & $38 / 7 / 0 / 0 / 0$ & $\mathrm{p}=0.169$ \\
\hline & Tooth vitality* & $64 / 2 / 0 / 0 / 0$ & $29 / 8 / 0 / 0 / 0$ & $\mathrm{p}=1.000$ \\
\hline
\end{tabular}

*20 endodontically treated teeth. 
not be an advantage if the examiner training to repair/ replacement decision-making by general dentists is based in reasons that may contradict the scientific evidence (19). The most common reason for repair or replacement of composite restorations is secondary caries (1). Nevertheless, general dentists can confuse marginal staining with marginal caries and prematurely replace those restorations. Contrariwise, in the Dental School environment, each detail and decision to repair or replace the restorations are widely discussed between the undergraduate student and the supervisor. Thus, even with the low experience on practice by the students, maybe they are more critical in the reliability of restoration replacement decision-making than the general dentists.

The present study showed lower annual survival rate for anterior restorations than for posterior ones, which corroborates previous finding (20). The mains causes for failures were different, being secondary caries and fracture for posterior restorations and fracture and aesthetic reason for anterior ones. Clinically, the superficial brightness, fracture and retention there were criteria with differences between anterior and posterior restorations. The anterior restorations showed less satisfactory superficial brightness than posterior. Superficial brightness is an aesthetic property and failures related to aesthetic qualities (as color, anatomical form, surface stain, brightness) are often reported when restorations are repaired/replaced for aesthetic reasons $(7,21)$. Moreover, it is important to highlight that the aesthetics aspects play a role in our modern society. Patients may request to have anterior restorations replaced because they do not like their aesthetics appearance, and this aspect could have an influence in the lower annual survival rate for anterior restorations.

In the present study the posterior restoration showed more fractures and loss than anterior restorations. The higher occurrence of failure of posterior restorations due to fracture has already been reported in other studies $(1,7,16)$. From median and long-term clinical studies some factors were shown to influence the survival of restorations, as the caries risk or other patient-related factors $(5,22)$, the operator variables $(8,19)$, and last, but not least, the localrelated factors as tooth type, tooth position and number of restored surfaces $(1,7)$. In the present study, the composite restorations that presented equal or more than 3 restored surfaces showed more chance to fail over time. It has been reported that restorations involving a larger number of surfaces are more likely to fail (3) mainly due to fractures as the loss of marginal ridges reduces the resistance of the tooth structure (23). Including the increased amount of restorative material, this may increase the risk for premature fatigue of the restoration-tooth complex, favouring the occurrence of fracture. Further, this process may be affected by oclusal risk factors.

There is still a concern that composite restorations are more susceptible to secondary caries, in high-risk patients (16). It proposes that the adhesive interface could influence the caries development. Other than fracture, secondary caries has been also reported as a common reason for restoration failure $(1,3,5)$. In this study, secondary caries was present. This could be justified by the fact that the sample is exclusively of patients from low-income and socioeconomic level. In poorer populations the prevalence of dental caries is expected to be high. In this present study, patients risk factors as income, caries activity and occlusal and caries risk were factors that influenced the survival rate. The socioeconomic level can affect restoration survival; with poorer individuals presenting a higher chance of restoration failure (24).

The adhesive interface seems more instable due to factors such as adhesive technique, adhesive materials, polymerization shrinkage, type of dental substrate (enamel/ dentin) and the quality of the hybrid layer can critically act towards adhesive failure in the interface, thus increasing the risk of secondary caries for composite restorations (25). Those aspects are being investigated in an attempt to improve the clinical performance of composite restorations. The adhesive procedures of composite restorations is technique-sensitive, and may lead to errors and operator related failures due to lack or insufficient training, since several steps must be followed, regarding particularities of tooth conditioning / adhesive systems, handling of composite material and optimal moisture control $(12,26)$. All the restorative treatments performed at the Dental School -UFSM- evaluated in this present study were performed used a two-step etch-and-rinse adhesive system (Adper Single Bond 2, 3M ESPE). A recent practice-based study showed that restorations performed with simplified adhesive systems (2-step etch-and-rinse and 1-step selfetch) presented a higher risk of failure \% than restorations performed with adhesives with bonding resin as a separate step (3-step etch-and-rinse and 2-step self-etch) (27). Thus, using a 3-step etch-and-rinse it could be expected a superior performance of the restorations.

Differences regarding the restorative materials were not observed in the present study, which is also showed in several previous studies $(5,22,26,28)$ where the composite resin material did not influence the long-term restoration survival. However, other studies $(1,7)$ reported a significant contribution of the material on survival rates. A recent systematic review (3) showed that annual failure rates after 10 years were nearly the same for compact-filed (2.2\%) and midway-filed $(2.3 \%)$ resin composites. Thus, maybe others variables, as the patient-related factors, could play 
a stronger role than the materials variables.

The present study design combined both dental records and clinical assessment of restorations, which is not usual in retrospective studies. This combined method can be considered as a benefit, since at the dental records there was no information referring to the clinical restoration's characteristics and types of failures, and therefore, the clinical restoration's assessment could inform it. Thus, in the records data were not possible to establish the reasons for restoration failure, as it was not noted in the records. Overall, dentists, leading to possible biases, often undertake this aspect. The reasons for failures were only taken during the clinical evaluations, where the mains causes for failures were secondary caries and fracture for posterior restorations and fracture and aesthetic reasons for anterior restorations.

Another aspect that should be highlighted is that few patients returned for clinical evaluations (30\%), which should be taking into account: as either they were motived patients or oppositely, they were patients that presented dental needs. Moreover, in Brasil is often that patients visit other dentists, and this may have happened with some patients in our study in between the Dental School visits. However, in an attempt to overcome this aspect, we included only patients that have been present for check-up or follow-up treatment in the Dental School (with at least 1 annual recall) and we exclude patients that reported dental interventions in other dentists. Moreover, patients that did not show up for visits may have visited other dentists. Another aspect to consider regarding the records data refers on having insufficient information provided in the dental files and in these cases, restoration survival might be overestimated.

Within the limits of this retrospective evaluation, the conclusion of the present study based on dental records and clinical assessments showed that after 8-year follow-up, the annual failure rate of restorations placed by dental students was $2.62 \%$. There was a difference in the comparisons between anterior and posterior restorations groups, with anterior restorations failing more than posterior. The patient's risk factors influenced the restorations survival.

\section{Resumo}

Este estudo retrospectivo comparou a taxa de falha anual (TFA), razões para falha e fatores influenciadores da sobrevida de restaurações posteriores e anteriores de resina composta realizadas por estudantes de graduação. As restaurações deveriam estar em oclusão, com pelo menos um dente adjacente, e os pacientes deveriam ter comparecido a pelo menos 1 consulta anual de retorno. A investigação foi realizada em 2 analises separadas: 1) dados odontológicos de prontuário eletrônico de pacientes ( $n=333$ restaurações) foram selecionados para avaliar os fatores influenciadores na sobrevida; 2) avaliação de 30 pacientes por exame clinico das restaurações $(n=123)$ para verificar as características clinicas e a distribuição do tipo de falha. Os dados foram submetidos a
Kaplan-Meier método, Log-rank teste, regressão de Cox e Teste Exato de Fisher $(p<0,05)$. Após o período de 8 anos, a TFA foi $2,62 \%$. Houve diferença entre restaurações anteriores e posteriores $(p=0,005)$, sendo que anteriores mostraram maior TFA. Os fatores de risco à carie $(p<0,001)$ e 0 número de superfícies restauradas $(p=0,010)$ influenciaram a sobrevida de restaurações. Brilho superficial $(p=0,029)$, fratura $(p=0,025)$ e retenção $(p=0,011)$ foram critérios clínicos com diferenças entre restaurações anteriores e posteriores. Renda $(p<0,001)$, atividade de cárie $(p<0,001)$, risco à cárie $(p<0,001)$ e risco oclusal $(p<0,001)$ também influenciaram a sobrevida. Após 8 anos, a TFA das restaurações realizadas por estudantes de graduação foram clinicamente aceitáveis e afetadas pelos fatores de risco associados ao pacientes e a posição do dente na arcada; restaurações anteriores falham mais do que posteriores.

\section{Acknowledgements}

Authors acknowledge the participants and staff members, who were fundamental in conducing this clinical study.

\section{References}

1. Da Rosa Rodolpho PA, Donassollo $T A$, Cenci MS, Loguércio $A D$, Moraes RR, Bronkhorst EM, et al. 22-Year clinical evaluation of the performance of two posterior composites with different filler characteristics. Dent Mater 201;27:955-263.

2. Pinto GdosS, Oliveira $U$, Romano $A R$, Schardosim LR, Bonow ML, Pacce $\mathrm{M}$, et al. Longevity of posterior restorations in primary teeth: results from a paediatric dental clinic. J Dent 2014;42:1248-1254.

3. Opdam NJ, van de Sande FH, Bronkhorst $E_{1}$ Cenci MS, Bottenberg $P$, Pallesen $U$, et al. Longevity of posterior composite restorations: a systematic review and meta-analysis. J Dent Res 2014;93:943-949.

4. Palotie U, Vehkalahti MM. Reasons for replacement of restorations: dentists' perceptions. Acta Odont Scand 2012;70:485-490.

5. van de Sande FH, Opdam NJ, Rodolpho PA, Correa MB, Demarco $\mathrm{FF}$, Cenci MS. Patient risk factors' influence on survival of posterior composites. J Dent Res 2013;92:78S-83S.

6. Opdam NJ, Bronkhorst EM, Cenci MS, Huysmans MS, Wilson NH. Age of failed restorations: a deceptive longevity parameter. J Dent 2011;39:225-230.

7. Baldissera RA, Corrêa MB, Schuch HS, Collares K, Nascimento GG, Jardim PS, et al. Are there universal restorative composites for anterior and posterior teeth? J Dent 2013;41:1027-1035.

8. Opdam NJ, Loomans BA, Roeters FJ, Bronkhorst EM. Five-year clinical performance of posterior resin composite restorations placed by dental students. J Dent 2004;32:379-383.

9. Collins CJ, Bryant RW, Hodge KLV. A clinical evaluation of posterior composite resin restorations: 8-year findings. J Dent 1998;26:311-317.

10. Ástvaldsdóttir A, Dagerhamn J, van Dijken JW, Naimi-Akbar A, Sandborgh-Englund G, Tranæus S, et al. Longevity of posterior resin composite restorations in adults. A systematic review. J Dent 2015;43:934-954.

11. Al-Samhan A, Al-Enezi $\mathrm{H}$, Alomari Q. Clinical evaluation of posterior resin composite restorations placed by dental students of Kuwait University. Med Princ Pract 2010;19:299-304.

12. Wilson $\mathrm{NH}$, Lynch $\mathrm{CD}$. The teaching of posterior resin composites: planning for the future based on 25 years of research. J Dent 2014;42:503-516.

13. Hickel R, Roulet JF, Bayne S, Heintze SD, Mjör IA, Peters M, et al. Recommendations for conducting controlled clinical studies of dental restorative materials. J Adhes Dent 2007;9:121-147.

14. Hickel R, Peschke A, Tyas M, Mjör I, Bayne S, Peters M, et al. FDI World Dental Federation - clinical criteria for the evaluation of direct and indirect restorations. Update and clinical examples. Clin Oral Invest 2010;14:349-366.

15. Vandenbroucke JP, von Elm E, Altman DG, Gøtzsche PC, Mulrow CD, Pocock SJ, et al. Strengthening the Reporting of Observational Studies in Epidemiology (STROBE): explanation and elaboration. Epidemiology 2007;18:805-835. 
16. Opdam NJ, Bronkhorst EM, Loomans BA, Huysmans MC. 12-year survival of composite vs. amalgam restorations. J Dent Res 2010;89:1063-1067.

17. Isokangas $P$, Alanen $P$, Tiekso J. The clinician's ability to identify caries risk subjects without saliva tests - a pilot study. Community Dent Oral Epidemiol 1993;21:8-10.

18. Pintado MR, Anderson GC, DeLong R, Douglas WH. Variation in tooth wear in young adults over a two-year period. J Prosthet Dent 1997;77:313-320.

19. McAndrew $R$, Chadwick $B$, Treasure ET. The influence of a short training program on the clinical examination of dental restorations. Oper Dent 2011;36:143-152.

20. Frese $C$, Schiller $P$, Staehle HJ, Wolff D. Recontouring teeth and closing diastemas with direct composite buildups: A 5-year follow-up. J Dent. 2013;41:979-985.

21. Demarco FF, Collares K, Coelho-de-Souza FH, Correa MB, Cenci MS, Moraes RR, et al. Anterior composite restorations: A systematic review on long-term survival and reasons for failure. Dent Mater 2015;31:1214-1224.

22. Pallesen $U$, van Djiken JW, Halken J, Hallonsten AL, Hoigaard R. Longevity of posterior resin composite restorations in permanent teeth in public dental health service: a prospective 8 years follow up. J Dent 2013;41:297-306.

23. Roumanas ED. The frequency of replacement of dental restorations may vary based on a number of variables, including type of material, size of the restoration, and caries risk of the patient. J Evid Based Dent Pract 2010;10:23-24

24. Correa MB, Peres MA, Peres KG, Horta BL, Barros AD, Demarco FF. Do socioeconomic determinants affect the quality of posterior dental restorations? A multilevel approach. J Dent 2013;41:960-967.

25. Moraschini V, Fai CK, Alto RM, Dos Santos GO. Amalgam and resin composite longevity of posterior restorations: A systematic review and meta-analysis. J Dent 2015;43:1043-1050.

26. van Dijken JW, Lindberg A. A 15-year randomized controlled study of a reduced shrinkage stress resin composite. Dent Mater 2015;31:11501158.

27. Collares K, Opdam NJM, Laske M, Bronkhorst EM, Demarco FF, Correa MB, Huysmans MCDNJM. Longevity of Anterior Composite Restorations in a General Dental Practice-Based Network. J Dent Res. 2017;96:10921099.

28. van Dijken JW, Pallesen U. A randomized 10-year prospective followup of Class II nanohybrid and conventional hybrid resin composite restorations. J Adhes Dent 2014;16:585-592.

Received March 14, 2018

Accepted July 30, 2018 